\title{
Globe
}

Revue internationale d'études québécoises

\section{L’américanisation de la langue française sur Internet? Quelques aspects de la terminologie officielle et de l'usage des internautes}

\section{Is French Being Americanized on the Internet? An Examination of Terminology and of the Linguistic Behavior of Internet Users}

\section{Beatrice Bagola}

Volume 7, numéro 2, 2004

Américanités francophones. Ancrages médiatiques, mises en perspective historiques et comparatistes

URI : https://id.erudit.org/iderudit/1000863ar

DOI : https://doi.org/10.7202/1000863ar

Aller au sommaire du numéro

Éditeur(s)

Globe, Revue internationale d'études québécoises

ISSN

1481-5869 (imprimé)

1923-8231 (numérique)

Découvrir la revue

Citer cet article

Bagola, B. (2004). L’américanisation de la langue française sur Internet? Quelques aspects de la terminologie officielle et de l'usage des internautes. Globe, 7(2), 101-124. https://doi.org/10.7202/1000863ar
Résumé de l'article

L'ordinateur et plus particulièrement Internet, ou la Toile, ont transformé les sociétés et les langues dans le monde entier. L'utilisation croissante de ces nouvelles technologies intensifie le contact avec la langue anglaise. Les influences qui en résultent entre autres sur le plan lexical conduisent les gouvernements en France et au Québec à intervenir dans le but de consolider la langue française sur le réseau mondial. Ceci contribue d'une part à établir une francisation, mais également, grâce aux différences dans la terminologie officielle, à créer une certaine hétérogénéité. Par ailleurs, la progression du français - et la régression simultanée de l'anglais - n'est pas seulement l'effet de la politique linguistique et de la vitalité des internautes francophones, mais aussi le résultat d'une sensibilité naturelle de leur part envers leur langue maternelle. Après examen de divers exemples, on peut dire que le comportement langagier des internautes contribue en quelque sorte à un enrichissement du français ainsi qu’à une certaine clarté de la langue. 


\title{
L'américanisation de la langue française sur Internet? Quelques aspects de la terminologie officielle et de l'usage des internautes
}

\author{
Beatrice Bagola \\ Université de Trier (Allemagne)
}

\begin{abstract}
Résumé - L'ordinateur et plus particulièrement Internet, ou la Toile, ont transformé les sociétés et les langues dans le monde entier. L'utilisation croissante de ces nouvelles technologies intensifie le contact avec la langue anglaise. Les influences qui en résultent entre autres sur le plan lexical conduisent les gouvernements en France et au Québec à intervenir dans le but de consolider la langue française sur le réseau mondial. Ceci contribue d'une part à établir une francisation, mais également, grâce aux différences dans la terminologie officielle, à créer une certaine hétérogénéité. Par ailleurs, la progression du français - et la régression simultanée de l'anglais - n'est pas seulement l'effet de la politique linguistique et de la vitalité des internautes francophones, mais aussi le résultat d'une sensibilité naturelle de leur part envers leur langue maternelle. Après examen de divers exemples, on peut dire que le comportement langagier des internautes contribue en quelque sorte à un enrichissement du français ainsi qu'à une certaine clarté de la langue.
\end{abstract}

Is French Being Americanized on the Internet? An Examination of Terminology and of the Linguistic Behavior of Internet Users.

Abstract - The computer and the World Wide Web in particular have had an important impact on societies and languages all over the world. The growing use of these new technologies and their linguistic consequences - among others on a lexical level - have led the governments of France and Quebec to decide on political measures to consolidate the French idiom where the www terminology is concerned. The advance of French - and the simultaneous decline of English on the Internet - is not only the effect of linguistic policy and the vitality of French speaking Internet users, but also the result of a natural sensitivity of these users towards their mother tongue. On the basis of various examples, it can be said that the linguistic bebaviour of Internet users leads not only to a certain lexical enrichment but also to a standardization of the French language.

Beatrice Bagola, - L'américanisation de la langue française sur Internet ? Quelques aspects de la terminologie officielle et de l'usage des internautes :, Globe. Revue internationale d'études québécoises, vol. 7, $\mathrm{n}^{\circ} 2,2004$. 
C'est à l'agence militaire américaine DARPA (Defense Advanced Research Project Agency) que le principe d'Internet, c'est-à-dire la transmission des informations par paquets à travers des lignes de communication électroniques, a vu le jour. En 1972, le système ARPAnet et celui du courriel ont été officiellement présentés. Ensuite, le programme Internetting a été développé, pour aboutir à Internet :

Origine: [Internet] abréviation de interconnection of networks. Le mot Internet a été créé en 1974 par Vinton Cerf et Robert Kahn lorsqu'ils ont mis au point le protocole de transmission TCP/IP. La première apparition de la forme abrégée Internet avec majuscule se trouve dans le développement de l'acronyme TCP/IP, à savoir Transmission Control Protocol/Internet Protocol. Le réseau Internet succéda alors au réseau ARPnet qui appartenait au ministère de la Défense américain ${ }^{1}$.

Internet a été de plus en plus utilisé aux États-Unis sous différentes formes d'application, par exemple le courriel et Telnet. Mais c'est surtout le Web (la Toile) qui a contribué au développement d'Internet comme nous le connaissons aujourd'hui. C'est en 1990 que Tim Berners-Lee, du CERN (Centre Européen de la Recherche Nucléaire) à Genève, a enrichi le système d'une composante importante : le World Wide Web. Internet, et la Toile plus particulièrement, ont transformé d'une manière impressionnante les sociétés dans le monde entier :

[Le Web] a permis l'adoption de l'Internet par le grand public. Son caractère convivial, sa simplicité d'utilisation et son interactivité en ont fait un vecteur de communication privilégié pour les organisations comme pour les utilisateurs de l'Internet ${ }^{2}$.

À ces aspects technologique et social d'Internet s'ajoutent des considérations politiques et économiques. Sur le plan politique, il s'agit entre autres de questions d'infrastructure, du financement dans les domaines de la recherche et de l'éducation, ainsi que dans les secteurs juridique,

1. Gabriel Otman, Les mots de la cyberculture, Paris, Belin, coll. "Le français retrouvé ", 1998, p. 183.

2. Arnaud Dufour et Solange Ghernaouti-Hélie, Internet, Paris, Presses universitaires de France, coll. "Que sais-je ? , 2002, p. 4. 
économique et linguistique. En ce qui concerne l'économie, on peut dire qu'en ce début de troisième millénaire une nouvelle économie - liée à l'a ancienne - - s'est établie ${ }^{3}$.

L'hégémonie commerciale et technologique américaine - historique et actuelle - continue de donner lieu à de nombreux débats sur le phénomène d'américanisation ${ }^{4}$. Dans le cas d'Internet, la question se pose également sur le plan linguistique. Cette technologie d'origine américaine, c'est-à-dire de langue anglaise, exerce en effet une influence linguistique sur les autres langues et cultures du monde.

Décrire les multiples relations entre Internet et la langue française, c'est tout d'abord tenir compte du rôle joué par des institutions officielles, mais c'est aussi prendre conscience de l'importance des internautes et de leur usage individuel de la langue. Comme on dispose ici d'un espace limité, on s'attachera, après quelques remarques préliminaires et des réflexions sur le français parmi les autres langues sur la Toile, à quelques aspects de la politique linguistique concernant Internet, et plus particulièrement aux efforts de standardisation lexicale. Le lexique, après tout, constitue en quelque sorte le miroir des changements linguistiques entraînés par cette nouvelle technologie, changements qui seront illustrés ici par différentes formes lexicales reflétant souvent les divergences entre l'usage officiel et l'usage des internautes.

\section{Le français et le plurilinguisme sur Internet}

La domination de l'anglais sur Internet et sur le marché a fait en sorte que d'autres langues "sont à la remorque de l'anglais ${ }^{5}$ " dans le but de s'assurer une présence sur la Toile. Le développement de ce média est pourtant de plus en plus marqué par des exigences d'internautes non an-

3. Ibid., p. 7.

4. On verra à ce sujet l'ouvrage d'Yvan Lamonde, Ni avec eux ni sans eux-le Québec et les États-Unis, Montréal, Nuit blanche éditeur, 1996, et le numéro de Politique et sociétés dirigé par Guy Lachapelle et Louis Balthazar (vol. 18, $\mathrm{n}^{\circ} 1$, - L'américanité du Québec •, 1999).

5. Jean-Claude Corbeil, I comme informatique, industrie de la langue et internet *, Bernard Cerquiglini, Jean-Claude Corbeil, Jean-Marie Klinkenberg et Benoît Peeters [éd.], Le français dans tous ses états, Paris, Flammarion, 2000, p. 128. 
glophones ainsi que par la productivité de ces locuteurs dans les secteurs public et privé, ce qui accroît la présence de langues autres que l'anglais. Le tableau à la page suivante montre le nombre d'internautes par langue (en millions) et la répartition des différentes langues sur Internet.

Le tableau relève une baisse et une croissance de l'anglais. Le pourcentage d'internautes anglophones a chuté, passant de $47 \%$ en 2000 à $35,2 \%$ en $2004^{6}$. L'accès Internet en anglais, par contre, croît continuellement. En 2000, le nombre d'anglophones connectés au réseau mondial était estimé à 192,1 millions. Aujourd'hui, il s'élève à 295,4 millions ${ }^{7}$. L'anglais demeure omniprésent, car les États-Unis devancent encore les autres pays entre autres dans le commerce électronique. Mais ce développement peut également être dû au fait qu'un certain nombre d'internautes non anglophones favorisent cet idiome au détriment de leur langue maternelle ${ }^{8}$. Au point de vue linguistique, toutefois, la tendance va de plus en plus contre cet unilinguisme:

If your primary task is building market awareness,
developing brands, and generating business, you are better
off regarding your target audiences as communities of
interest shaped by national - ratber than global -
characteristics. Most non-English Internet users prefer
websites in their own language, according to IDC's eWorld
2001 Survey. Almost $34 \%$ of French respondents prefer to
visit websites in English, while $62 \%$ prefer sites in French 9.

6. Xavier Bihan, "La fin de l'hégémonie de l'anglais sur l'internet [sic]?" Fremdsprachenunterricht, $\mathrm{n}^{\circ} 44,45 ; 2000$, p. 459-462; Commission des états généraux sur la situation et l'avenir de la langue française au Québec 2001, Le français, une langue pour tout le monde: une nouvelle approche stratégique et citoyenne; Jean-Claude Corbeil, op. cit., p. 125-137; Languages On The I-net/Global Internet Statistics, www. glreach.com, 30 septembre 2002.

7. Languages On The I-net/Global Internet Statistics, www.glreach.com/ globstats/index.php3, 29 janvier 2005.

8. www.global-reach.biz/eng/index.php3, 21 janvier 2005.

9. www.global-reach.biz/eng/index.php3, "Si votre tâche première est de susciter l'attention du marché, de développer des marques et de favoriser le commerce, il vaudra mieux considérer vos diverses audiences comme autant de communautés d'intérêt ayant des caractéristiques nationales plutôt que globales. Selon un sondage ICD eWorld de 2001, la plupart des internautes non anglophones préfèrent des sites dans leur langue. Presque $34 \%$ des répondants francophones affirment préférer des sites anglais, alors que $62 \%$ préfèrent des sites français " [notre traduction], 21 janvier 2005. Voir aussi le tableau 1. 


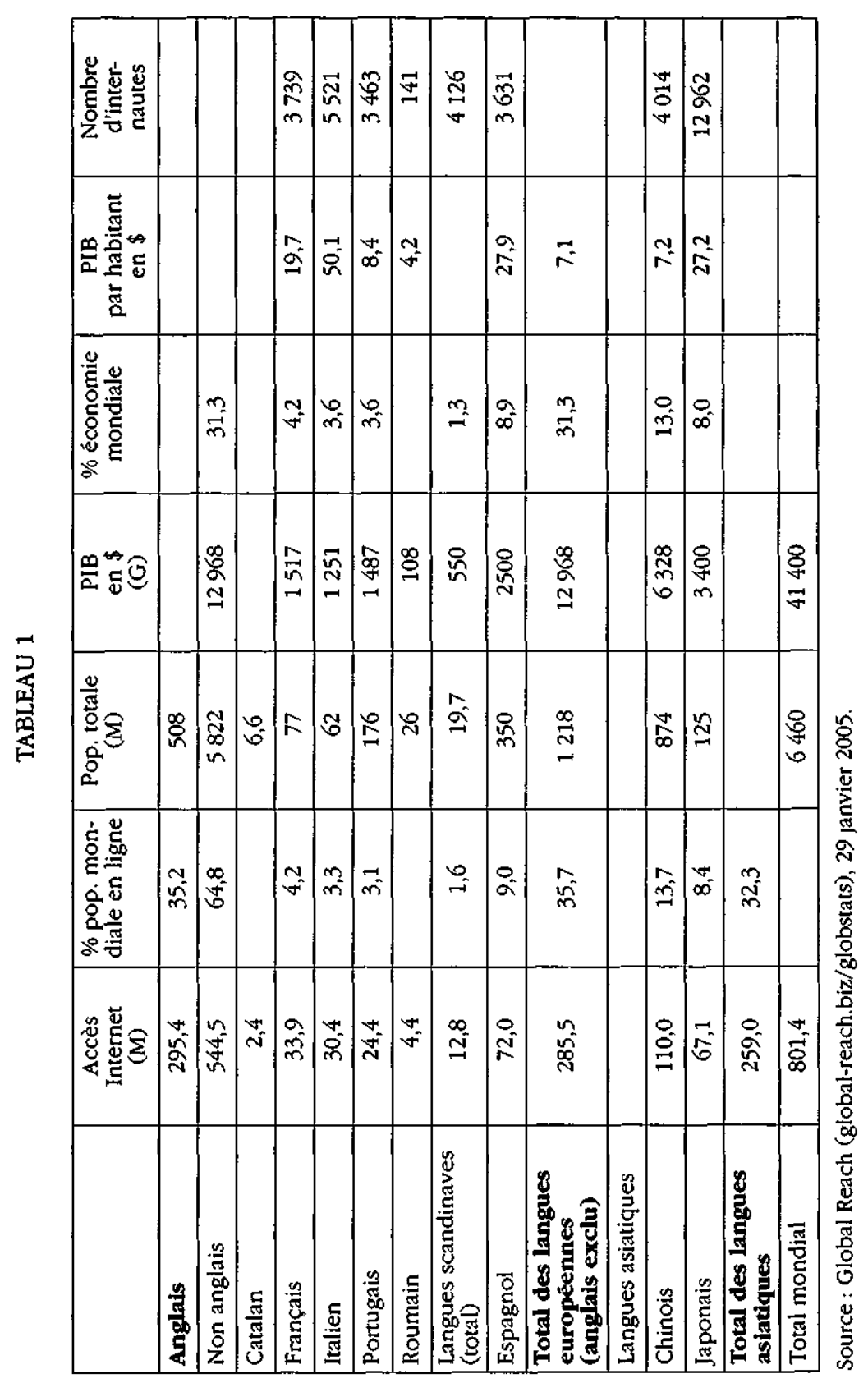


Ainsi, la diversification linguistique et culturelle a augmenté par rapport à l'enquête effectuée par Global Reach en décembre $2000^{10}$. Il faut surtout remarquer que l'accès des internautes non anglophones a plus que doublé, passant de 211,3 millions en 2000 à 544,5 millions en 2004, dont 285,5 millions se situent en Europe (voir tableau 1). Le tableau 1 souligne par ailleurs l'existence d'un certain nombre de langues romanes dans le plurilinguisme sur Internet. Grâce aux nouveaux internautes, les francophones passent de 14,2 millions $(3,5 \%)$ à 33,9 millions $(4,2 \%)$.

L'espace limité ne nous permet pas de discuter le développement d'autres langues romanes ou des langues asiatiques sur la Toile. Ce multilinguisme avait été prédit par Michel Bélair, qui constatait en février 1999 que "l'anglais régresse sur Internet et [que] si la tendance se maintient, l'anglais n'occupera plus en 2002 que le tiers du territoire de l'inforoute planétaire. L'heure est au multilinguisme ${ }^{11}$ ".

Ces statistiques ne contiennent pas de données quant au nombre d'internautes branchés à partir des différents pays. Après recherche, on constate des divergences considérables entre les différentes études. Ainsi, le Journal du Net estimait la population française branchée en décembre 2002 à 18,7 millions, et en mars 2003 à 20,3 millions. Dans un article du journal Le Monde, Cécile Ducourtieux parle quant à elle de "seize millions de Français ${ }^{12}$ "branchés en juin 2002, pour préciser ensuite que " [s]euls les plus de 50 ans résistent. Alors qu'ils constituent $40 \%$ de la population, ils ne représentent que $15 \%$ du total des internautes français *. Les sites sont visités entre autres pour obtenir des informations, utiliser gratuitement un service de courriel, ou encore à des fins de recherche, d'affaires, de finance et d'économie, de divertissement et de communication. Concernant les statistiques de la seconde puissance francophone, le Québec, on constate que " $88 \%$ des adolescents québécois (de 12-17 ans) et plus de 3 millions d'internautes québécois(es) âgé(es) de 18 ans ou plus utilisent l'Internet sur une base

10. Languages On The I-net/Global Internet Statistics, décembre 2000.

11. Michel Bélair, "L'anglais régresse sur internet ", Le Devoir, 25 février 1999, p. 1.

12. Cécile Ducourtieux, "Un tiers des Français familiarisés avec Internet ", Le Monde-Sélection bebdomadaire, 14 septembre 2002, p. 5. 
régulière ${ }^{13}$. En général, les jeunes consacrent beaucoup de temps au clavardage.

Malgré ces divergences statistiques, il est incontestable que les internautes branchés dans une langue autre que l'anglais occupent aujourd'hui une place considérable sur Internet et que ce nombre continue d'augmenter. La progression des langues romanes - nationales (français, espagnol), régionales ou minoritaires (catalan) - témoigne de la vitalité de ce média et de l'expansion de son marché en langue maternelle autre que l'anglais ${ }^{14}$. Une évolution qui se base non seulement sur le comportement des internautes, mais aussi sur une politique linguistique et culturelle de certains pays comme la France ou le Québec qui vise à faire du français un idiome puissant sur Internet.

Cependant, au niveau international, le français ne constitue pas une langue homogène, présentant plutôt plusieurs variêtés.

\section{Concurrence du français avec l'anglais. La terminologie officielle et l'usage des internautes}

La politique linguistique d'organismes tels que l'Agence internationale de la Francophonie ou le Conseil international francophone des langues (CIFLA) est axée à la fois sur la promotion des langues nationales ou parlées dans les pays francophones et sur l'utilisation du français dans la nouvelle technologie ${ }^{15}$. Parmi les nombreuses actions officielles à l'intérieur de la francophonie, on remarque par exemple le

13. www.infometre.cefrio.qc.ca. Voir aussi l'article de Céline Roquette, * Un tiers des adultes ont déjā utilisé l'Internet •, www.insee.fr, 2002 ; et le texte de Xavier Bihan, "L'enjeu linguistique de l'Internet pour l'espace francophone ", Kerstin Störl et Johannes Klare [êd.], Romanische Spracben in Amerika. Festscbrift für Hans-Dieter Paufler zum 65, Francfort et New York, Lang, 2002, p. 87-97 ; ainsi que l'entrée * Internet * dans l'encyclopédie de l'Agora, www.agora.qc.ca.

14. Jean-Claude Corbeil, op. cit., p. 129.

15. Agence internationale de la Francophonie, 2000. Voir aussi Roberto Mann, - Frankophonie im Internet ", Ingo Kolboom, Thomas Kotschi et Edward Reichel [éd.], Handbuch Französiscb. Sprache Literatur Kultur Gesellschaft. Für Studium, Berlin, Erich Schmid Verlag Lehre, coll. " Praxis ", 2002, p. 473b-476b. 
programme "Contenu numérique européen sur les réseaux mondiaux (eContent) ", adopté par le Conseil le 22 décembre 2000, pour une durée de quatre ans. Mais c'est surtout sur le plan des approches nationales que la politique linguistique des différents pays est la plus sensible, parce qu'il a est indispensable d'enrichir le français pour lui permettre de disposer de mots exprimant toutes les notions du monde contemporain et de veiller à ce qu'il soit présent dans les nouvelles technologies de l'information et de la communications ${ }^{16}$. .

En France, les liens entre la langue et l'État sont étroits. La politique linguistique remonte au $\mathrm{xVI}^{\mathrm{e}}$ siècle lorsque l'ordonnance de VillersCotterêts (1539) impose le français comme langue juridique. Alors que des auteurs comme Vaugelas contribuent à la codification de la langue, l'Académie française, fondée en 1635 comme organisme de l'État, reçoit comme mission de donner des "règles certaines "à la langue française. La circulaire 72 du 28 prairial, an II (1794) reconnaitt le français comme langue d'État ${ }^{17}$.

Le contact avec la langue anglaise se manifeste à partir du XviIr siècle. L'influence de l'anglais, qu'il soit britannique ou nord-américain, atteint son paroxysme dans les années 1950 et 1960. L'American way of life devient vite le "symbole" de la deuxième moitié du $\mathrm{xx}^{\mathrm{e}}$ siècle ${ }^{18}$. Deux grandes lois linguistiques seront votées en faveur du français, interdisant l'utilisation des termes étrangers " dont il existe en français un équivalent agréé19 " : la loi Bas-Lauriol (1975) et la loi Toubon (1994).

On aurait pu s'attendre, compte tenu du contact permanent et massif avec l'anglais sur Internet, à des réactions fortes de la France et de ses organismes dès le début. Curieusement, dans ce pays où la protection de la langue est une préoccupation depuis le $\mathrm{xvi}^{\mathrm{e}}$ siècle, Internet est resté

16. www.relais-culture-europe.org/programme/soc_info/e_content/ xe_content.pdf, 21 janvier 2005 ; Le français et le plurilinguisme dans la société de l'information, www.culture.fr/culture/dglf/, septembre 2002.

17. Mireille Huchon, Histoire de la langue française, Paris, Le Livre de poche, coll. * Références Littérature ", 2002, p. 205.

18. Claude Hagège, Le français, bistoire d'un combat, Paris, Michel Hagège, 1996, p. 111.

19. Ibid., p. 151. 
relativement longtemps ignoré par les autorités, si on compare à d'autres pays francophones d'Europe ou encore au Québec ${ }^{20}$.

L'entrée de la France sur la Toile est considérée par le gouvernement, depuis 1996, comme un enjeu décisif pour l'avenir. Constatant le nombre croissant d'internautes français, le gouvernement a déclaré que - les enjeux linguistiques liés aux nouvelles technologies de l'information et de la communication, en particulier l'Internet, constituent une préoccupation du gouvernement ${ }^{21}$. Les efforts du gouvernement ont pour but de promouvoir le français sur Internet et de faire en sorte qu'il devienne la langue de communication privilégiée sur le réseau. Afin d'améliorer la position de l'idiome national à l'intérieur de la société de l'information, le gouvernement français a lancé en 1998 le Programme d'action gouvernemental pour l'entrée de la France dans la société de l'information (PAGSI). Paru après un an de travail, le Rapport au Parlement de la Délégation générale à la langue française et aux langues de France (DGLFLF) mentionne que

[le Programme] a commencé à porter ses fruits et [que] notre pays est en voie de combler le retard qu'il connaissait dans le développement et l'usage des technologies de l'information et de la communication [...]. Cependant, le français n'occupe pas encore sur les réseaux la place de grande langue de communication internationale qui pourrait lui revenir ${ }^{22}$.

Les actions massives en faveur du français portent entre autres sur la diffusion des données publiques, sur l'établissement de termes français pour la société de l'information, sur le développement des outils d'accès à l'information et de communication, et enfin sur la création de ressources linguistiques pour développer le traitement automatisé de l'information. La Délégation générale à la langue française et aux langues de France contribue par ailleurs à identifier l'ensemble des enjeux linguistiques de la société de l'information à l'échelle nationale, européenne,

20. Xavier Bihan, op. cit., 2002.

21. Délégation générale à la langue française et aux langues de France, Rapport au Parlement, 1999, p. 107.

22. Ibid. 
francophone et internationale. Un article tiré du Monde bebdomadaire en 2002 résume en quelque sorte le chemin parcouru en France :

Cinq ans jour pour jour après le "discours d'Hourtin " où Lionel Jospin, premier ministre, lança officiellement l'" internétisation " de la France, les chiffres de connexion parlent d'eux-mêmes. [...] Entre 2000 et mi-2002, le nombre d'internautes a continué de crôtre régulièrement [...]. Seize millions de Français sont connectés en juin 2002. "Nous ne sommes pas loin du phénomène de masse n, affirme Louis Rougier. [...]. L'usage d'Internet est par ailleurs devenu quotidien pour un tiers des internautes ${ }^{23}$.

D'autre part, le plan RE/SO 2007 (Pour une RÉpublique numérique dans la SOciété de l'information)

vise, comme l'a souligné Jean-Pierre Raffarin, à construire et favoriser "une République numérique, fidèle à la devise qui est au fronton de nos institutions ". "Liberté, égalité, fraternité " doivent ainsi pouvoir s'" ancrer dans la société de l'information ${ }^{24}$ ".

Le but de ces efforts législatifs en faveur du commerce et de l'avenir du monde virtuel est de convaincre les internautes français des bénéfices du monde virtuel pour assurer à la France "la position de leader européen dans la société de l'information ${ }^{25}$.

Cette politique et les discussions sur le statut et le prestige de la langue française sur Internet concernent aussi les usages linguistiques ${ }^{26}$. Ce qu'il faut noter ici, c'est la différence entre la diffusion de la langue française sur le réseau mondial et sa standardisation. Alors que la diffu-

23. Cécile Ducourtieux, op. cit.

24. http://www.telecom.gouv.fr/internet/index_len.htm, 11 décembre 2002.

25. lbid.

26. Voir Christian Ossenkop, "Die Varianten des gegenwärtigen Französisch *, Ingo Kolboom, Thomas Kotschi et Edward Reichel [éd.], Handbuch Französisch. Spracbe Literatur Kultur Gesellschaft. Für Studium, Berlin, Erich Schmid Verlag Lehre, coll. * Praxis *, p. 68a-77b et Mireille Huchon, op. cit. 
sion porte sur la langue en général, la standardisation ou les normes linguistiques concernent avant tout l'usage lexical.

Le contact permanent avec l'anglais, ou plus exactement l'angloaméricain, langue principale de cette nouvelle technologie, influence la langue française. Différents groupes - la Commission spécialisée de terminologie et de néologie de l'informatique et des composants électroniques (CSTIC), la Commission spécialisée de terminologie et de néologie des télécommunications (CSTNT), le Groupe d'experts français pour les standards d'Internet (GFSI), la Délégation générale à la langue française et aux langues de France (DGLFLF), la Commission générale de terminologie et de néologie et l'Académie française - travaillent à l'établissement d'une terminologie française d'Internet, conformément à la loi du 4 août 1994 relative à l'emploi de la langue française et au décret $n^{\circ}$ 96-602 du 3 juillet 1996 pour le monde du travail, le réseau et d'autres domaines reliés. Ils œuvrent également à la consolidation de la présence du français sur Internet ${ }^{27}$.

Il existe actuellement un bon nombre de dictionnaires et de glossaires en ligne concernant la terminologie d'Internet, parmi lesquels se trouvent les banques de données officielles de la DGLFLF. Les termes officiels

ne peuvent être publiés au Journal officiel [...] que si elle [l'Académie française] a formulé un avis favorable [...]. C'est la première fois que l'Académie française se trouve associée officiellement et de façon systématique aux travaux institutionnels de terminologie et de néologie ${ }^{28}$.

La Délégation générale à la langue française et aux langues de France met à la disposition des internautes les listes de termes publiées au Journal officiel par la Commission générale de terminologie. Une première liste de termes de base a été établie et publiée au Journal officiel,

27. http://www.culture.gouv.fr/culture/dglf/2002, 21 janvier 2005.

28. Isabelle Bouret, "L'État et l'enrichissement de la langue française *, http://www.geocities.com/Eureka/Office/1936/terminol.html, 2000, p. 2. 
après avis de l'Académie française, le 16 mars $1999^{29}$. Les listes sont continuellement actualisées.

Le résultat des travaux normatifs consacrés à la codification de la langue est en quelque sorte le bon usage d'Internet en France. L'utilisation courante des termes officiels dépend de l'usage commun sur Internet. Une des cibles d'une telle politique est par conséquent l'internaute. Celui-ci est, selon Ipsos-Médiangles, "un individu qui s'est personnellement connecté au Web au moins une fois au cours des trente derniers jours à partir de son domicile, lieu de travail, école ou université ${ }^{30}$. .

Grâce à ce bon usage, l'internaute en France peut recourir à des termes français au lieu d'utiliser les expressions anglaises. Mais à quel point l'internaute se sert-il de cette terminologie officielle? Le tableau suivant donne une idée des usages sur la Toile.

TABLEAU 2

\begin{tabular}{|l|l|}
\hline \multicolumn{1}{|c|}{ Terminologie officielle } & \multicolumn{1}{c|}{ Usage des internautes } \\
\hline autoroutes de l'information & autoroutes de l'information \\
\hline barrière de sécurité & firewall \\
\hline pare-feu & pare-feu \\
\hline causette & chat, tchatche \\
\hline forum & forum \\
\hline frimousse & smiley \\
\hline Internet & internet, Internet, net \\
\hline $\begin{array}{l}\text { page d'accueil } \\
\text { page sur la Toile } \\
\text { serveur } \\
\text { Toile d'araignée mondiale (T.A.M.), } \\
\text { Toile mondiale, Toile }\end{array}$ & $\begin{array}{l}\text { page d'accueil } \\
\text { page sur la Toile } \\
\text { serveur } \\
\text { Web, w3, www, ouèbe }\end{array}$ \\
\hline $\begin{array}{l}\text { foire aux questions, questions } \\
\text { courantes }\end{array}$ & faq/FAQ \\
\hline
\end{tabular}

29. DGLFLF, Rapport au Parlement, op. cit., p. 123.

30. Cécile Ducourtieux, op. cit. 
Des lexèmes tels que "autoroutes de l'information " (information bighway, information superbigbway), "page d'accueil " (home page), " page sur la Toile - (webpage, web page), "serveur "(server, on-line data service), "forum " (newsgroup), "Internet "(Internet network, Internet, Net) sont aujourd'hui fort répandus sur la Toile française. En ce qui concerne le terme "Internet ", dérivé de l'américain pour * internetworking " (inter- et network), son emploi peut peut-être s'expliquer par le fait que la terminologie officielle reprend parfois des termes déjà répandus parmi les internautes ${ }^{31}$.

Comme on peut le constater, la formation des mots et l'intégration du vocabulaire de cette nouvelle technologie américaine suivent les règles du français. Les termes français "Internet ", "Web " et " chat ", entre autres, font partie de ceux qui correspondent aux formes anglaises. $\grave{A}$ côté de cette adaptation graphique, il existe d'une part des calques comme "autoroutes de l'information " et "page sur la Toile ", et d'autre part des mots français enrichis sur le plan sémantique, tels que "causette ", "forum ", "serveur " et "Toile ". Dans le cas de "page d'accueil ", l'emprunt fait référence à la définition de "bome page ", qui insiste sur sa fonction d'introduction: " an individual's organization's introductory document on the World Wide Web ${ }^{32}$. Les noms composés suivent l'ordre des mots français : le déterminé précède le déterminant, ce qui n'est pas le cas dans la langue anglaise.

Par ailleurs, les néologismes français peuvent ensuite produire des dérivés ou des composés. Citons comme exemple les préfixes tels "cyber " ou "e- " (électronique ${ }^{33}$ ), qui sont lexicalisés. On rencontre ainsi des formes comme "cyber-café(s) ", "cyberculture ", "cyberespace " et " cybernaute ${ }^{34}$ "(la terminologie officielle ajoute à l'entrée * internaute *le commentaire suivant: " on rencontre aussi le terme "cybernaute 35 " "). L'utilisation du préfixe "e- " (electronic) est aussi très répandue, formant

31. Petra Brasselmann, - Sprachpolitik und Sprachbewußtsein in Frankreich heute ", Romanistische Arbeitsheft, Tübingen, Niemeyer, 1999.

32. Compact Oxford English Dictionary.

33. Le Petit Robert, 2002.

34. Ibid. Voir aussi Cécile Ducourtieux, op. cit.

35. http://www.culture.gouv.fr/culture/dglf/cogeter/16-03-99-internet-listes.html, 29 janvier 2005. 
des composés comme "ePolitique ", "eSélection ", "eDémocratie ", "ecommerce", "e-administration", "e-ministère", entre autres dans les médias, le commerce et l'administration. On constate dans ce cas-ci une graphie variable, phénomène bien connu dans le contexte d'Internet. D'autre part, parmi les néologismes, on trouve aussi " internétisation ${ }^{36}$ " et "Webisation ${ }^{37}$. Le vocabulaire de la Toile française comprend également un grand nombre d'abréviations, de sigles et d'acronymes ${ }^{38}$. Enfin, un autre trait caractéristique est l'existence d'un certain nombre de synonymes officiels pour un même terme anglais, comme "barrière de sécurité " et "pare-feu " pour firewall; "diffusion systématique sur la Toile ", "diffusion réticulaire systématique ", "diffusion sur la Toile " et " diffusion réticulaire "pour webcasting ou netcasting, ou encore, "foire aux questions ", "fichier des questions courantes " et "questions courantes " pour frequently asked questions.

Bien que l'usage du français gagne en prestige au détriment de l'anglais en France, on s'aperçoit que la langue d'un grand nombre d'utilisateurs de la Toile s'écarte de cette norme, car si des anglicismes ont cédé leur place aux termes officiels français dans le quotidien, les termes anglais ont été, eux, maintenus dans un grand nombre de sites. L'influence de l'anglais (voir tableau 2) est encore évidente en France. Des moteurs de recherche comme Yahoo et Google, ainsi que d'autres sites français témoignent de ce "mauvais usage ». En naviguant sur Internet, on trouve ces formes concurrentes:

36. Cécile Ducourtieux, op. cit.

37. http://www.systematic-lorraine.net/cgi/site/xsh/page, 29 janvier 2005.

38. Voir Henriette Walter, Honni soit qui mal y pense. L'incroyable bistoire d'amour entre le français et l'anglais, Paris, Robert Laffont, 2001 et Petra Brasselmann, op. cit. 
TABLEAU 3

\begin{tabular}{|c|c|c|c|c|c|c|}
\hline Terme & $\begin{array}{l}\text { Toile : } \\
\text { Total }\end{array}$ & $\begin{array}{l}\text { Toile : } \\
\text { Total }\end{array}$ & $\begin{array}{l}\text { Pages } \\
\text { franco- } \\
\text { phones }\end{array}$ & $\begin{array}{l}\text { Pages de } \\
\text { la France }\end{array}$ & $\begin{array}{l}\text { Pages du } \\
\text { Canada }\end{array}$ & \\
\hline & 2002 & 2005 & 2005 & 2005 & 2005 & \\
\hline firewall & 71800 & 28900000 & 1430000 & 1410000 & 760000 & \\
\hline $\begin{array}{l}\text { pare feu (terme } \\
\text { officiel) }\end{array}$ & 12200 & 561000 & 528000 & 81000 & 40000 & (a) \\
\hline $\begin{array}{l}\text { pare-feu (terme } \\
\text { officiel) }\end{array}$ & & 439000 & 439000 & 320000 & 23000 & \\
\hline Web & 1690000 & 1030000 & 22100000 & 19900000 & 22600000 & \\
\hline ouèbe & $\cdots$ & 43300 & 41300 & 32000 & 837 & (b) \\
\hline wouebe & $\ldots$ & 282 & 260 & 193 & 70 & \\
\hline $\begin{array}{l}\text { Toile (terme } \\
\text { officiel) }\end{array}$ & 116000 & 1830000 & 1720000 & 1250000 & 722000 & (c) \\
\hline chat & 1080000 & 206000 & 15600000 & 13600000 & 10700000 & (d) \\
\hline $\begin{array}{l}\text { causette (terme } \\
\text { officiel) }\end{array}$ & & 31400 & 29000 & 18900 & & \\
\hline tcbatcb & 86000 & 741000 & 513000 & 266000 & 2630 & \\
\hline smiley & 42000 & 5360000 & 423000 & 414000 & 200000 & \\
\hline $\begin{array}{l}\text { frimousse (terme } \\
\text { officiel) }\end{array}$ & 5200 & 102000 & 91200 & 82000 & 5520 & \\
\hline $\begin{array}{l}\text { frequently asked } \\
\text { questions }\end{array}$ & 19500 & 26600000 & 132000 & 198000 & 150 & \\
\hline $\begin{array}{l}\text { foire aux } \\
\text { questions }\end{array}$ & 71700 & 1250000 & 1230000 & 580000 & 702000 & \\
\hline $\begin{array}{l}\text { questions } \\
\text { fréquemment } \\
\text { posées }\end{array}$ & 39700 & 246000 & 241000 & 121000 & 48800 & \\
\hline
\end{tabular}

(a) Les pages incluent aussi celles offrant une protection contre l'incendie.

(b) Pour l'année 2003 le nombre s'élève à 14500 occurrences.

(c) Il s'agit surtout de pages se référant au web.

(d) Ce chiffre comprend aussi un nombre considérable de sites concernant l'animal. 
Attachons-nous à titre d'exemple aux termes "firewall", "Web" et " chat". Dans le cas de "firewall", on a accès à des sites en anglais qui font très souvent référence aux logiciels. Parfois, une traduction est proposée à l'internaute. Le terme " pare-feu " conduit à des sites en français où l'on trouve régulièrement le synonyme "firewall". Voici un exemple typique de tels sites:

Firewall: [...] Firewall/Pare-feu. [...] Il existe de nombreuses manières de structurer un réseau pour protéger des systèmes à l'aide d'un pare-feu ${ }^{39}$. [...]

Mais l'utilisation de termes anglais ne se limite pas au domaine d'Internet : ils sont également répandus dans la presse, les autres médias et les manuels. Dans l'article cité plus haut, le terme "Web* est courant. La graphie signale deux variétés, une comme nom propre avec $\mathrm{W}$ majuscule, et l'autre avec w minuscule. "Web, web (World Wide Web)" est bien lexicalisé, entre autres dans Le petit Robert 2002. Le chat constitue un cas analogue. Le lexème "causette" (familier) prescrit par le bon usage est en concurrence avec le terme "chat ", qui fait partie de l'usage à l'intérieur et à l'extérieur du monde virtuel. En consultant Le petit Robert 2002, on trouve l'entrée chat avec une référence à la recommandation officielle "causette" $(409 \mathrm{~b})$, contrairement à l'entrée pour le lemme "Web". Nous reviendrons plus tard sur les termes "ouèbe " et "tchatche".

On le voit, au lieu de la clarté et de la cohérence exigées par la politique linguistique, l'usage témoigne plutôt de l'hétérogénéité linguistique d'Internet et de la Toile en France, qui est favorisée non seulement par la présence des anglicismes, mais aussi par des divergences à l'intérieur de la francophonie. Ces divergences se manifestent tout particulièrement par rapport aux terminologies officielles du Québec et de la France.

39. www.lifl.fr/ -boulet/formation/syst-dist/exposes2000-2001/firewall/ firewall.htm, 21 janvier 2005. 


\section{Concurrence à l'intérieur de la francophonie : France-Québec}

L'histoire de ces deux variantes est caractérisée par une politique linguistique restrictive et par des tendances puristes. La question de la défense de la langue au Québec se manifeste dès la cession de la Nouvelle-France à l'Angleterre, en 1763. L'anglais est alors la nouvelle langue d'État. La plupart des liens avec la France sont interrompus, et la langue française perd son statut de langue officielle. À la suite de ces changements politiques et linguistiques,

[...] la population française du Canada a réclamé le droit de continuer à faire usage de la langue française, le droit de conserver le droit civil français en lieu et place de la Common Law imposée au lendemain de la Conquête et le droit de pratiquer la religion catholique, face au protestantisme officiel des Britanniques. Ces droits ont été accordés aux Canadiens par l'Acte de Québec en 1774 et confirmés par l'Acte constitutionnel de $1791^{40}$.

Après l'adoption de la confédération canadienne, en 1867, le français et l'anglais sont reconnus à l'Assemblée nationale et dans les tribunaux par l'article 133 de la Constitution.

Exposé à l'anglais pendant de nombreuses années, le français finit par accueillir un grand nombre d'anglicismes dans son lexique. Claude Poirier résume ainsi ce processus :

En raison de son isolement linguistique sur le continent nord-américain et de son cheminement politique difficile, le Québec a de l'anglicisme une expérience tout à fait particulière : l'emprunt linguistique à l'anglais s'est en effet accompagné d'une anglicisation forcée du milieu de vie, touchant tour à tour les institutions politiques, le monde du travail, celui de la finance, des communications, des technologies, etc. Pour cette

40. http://www.spl.gouv.qc.ca/langue/tablematiere2.html, 21 janvier 2005. 


\section{REVUE INTERNATIONALE D'ÉTUDES QUÉBÉCOISES}

raison, les Québécois ont toujours appréhendé le phénomène linguistique de l'anglicisme à travers leur vision de francophones dominés et en ont développé une conception que Chantal Bouchard (1989) a bien caractérisée [...] " une obsession nationale : l'anglicisme "41.

C'est au XIX siècle que se font entendre les premières demandes des puristes québécois francophones, qui réclament des mesures de protection de la langue française. Un siècle plus tard, non seulement la discussion sur la pureté de la langue et de sa norme est au centre de l'intérêt de toute une société, mais elle est définitivement reprise par l'État. Au Québec comme en France, un lien direct existe entre la langue et la culture françaises, mais les Québécois - en contact permanent et direct avec le monde anglophone - défendent leur langue d'abord pour assurer sa simple survie.

Une telle protection est mise en place lors des changements politiques qui surviennent pendant la Révolution tranquille. Une politique linguistique s'établit, des lois - comme la loi 63 (1969), qui vise à promouvoir la langue française au Québec, et la Loi sur la langue officielle (loi 22, 1974) - sont votées. Avec l'adoption de la Charte de la langue française (1977), appelée aussi Loi 101, le français devient l'unique langue officielle du Québec. La succession des lois et les modifications de la Charte témoignent du combat décisif et efficace en faveur d'un aménagement du français et de sa diffusion aux niveaux national et international ${ }^{42}$.

Nous avons mentionné plus haut que la France affichait un certain retard quant à la politique d'Internet. Au Québec, au contraire, on

41. Claude Poirier [éd.], Anglicisme et identité québécoise. Séminaire interdisciplinaire. GPL 64536-GPL 64537, hiver 1992, résumés des conférences et notes de cours, Québec, Université Laval, p. 6.

42. La loi la plus controversée a été la loi 178 (modifiant la Charte de la langue française, en 1988), qui concernait la langue de l'affichage et l'unilinguisme français. Puis ont suivi la loi 86 (modifiant la Charte de la langue française, en 1993), la loi 40 (modifiant la Charte de la langue française, en 1997), la loi 171 (modifiant la Charte de la langue française, en 2000) et le projet de loi 104 (modifiant la Charte de la langue française, en 2002) (http://www.tlfq.ulaval.ca/axl/amnord/ quebecmodif.html ; http://www.olf.gouv.qc.ca/charte/reperes/reperes.html). 
adoptait en novembre 1992 une politique d'utilisation du français dans les technologies de l'information. Rappelant, en préambule, que ces technologies sont largement utilisées pour l'administration des programmes et pour les communications avec les citoyens, le gouvernement veut ainsi jouer un rôle déterminant en matière de promotion et d'utilisation du français dans ce domaine ${ }^{43}[. .$.$] .$

Le but officiel est en quelque sorte le même qu'en France : il s'agit avant tout d'établir et de promouvoir une politique d'utilisation du français dans les technologies de l'information.

L'utilisation de l'anglais dans les technologies de l'information appauvrit la langue et la culture françaises. Le langage informatique est surtout conçu, développé et commercialisé en langue anglaise et, par le fait même, l'anglais devient la langue de traitement des données et des textes. [...] Le gouvernement doit jouer un rôle déterminant en matière de promotion et d'utilisation du français dans les technologies de l'information. En effet, en se dotant d'une politique dans le domaine, le gouvernement reconnaît ainsi toute la portée économique, sociale et culturelle du français à travers les technologies de l'information. Il démontre également une volonté ferme de faire une place au français dans les technologies. Cette prise de position doit inciter le secteur privé à emboîter le pas, notamment en stimulant l'offre de produits informatiques en français [...]. La présente politique vise à généraliser l'utilisation du français dans les technologies de l'information comme langue de conception, d'utilisation, de diffusion et de formation ${ }^{44}$.

Une partie essentielle de cette politique est donc consacrée à la diffusion du français et à son utilisation dans les nouvelles technologies.

43. Commission des états généraux sur la situation et l'avenir de la langue française au Québec, Le français, une langue pour tout le monde. Une nouvelle approche stratégique et citoyenne, Montréal, 2001, p. 145.

44. http://www.tresor.gouv.qc.ca/doc/polifran.htm, 21 janvier 2005. 
Des institutions et des commissions travaillent également à l'établissement d'une terminologie officielle. Il convient de mentionner entre autres le rôle des commissions terminologiques et celui de l'Office québécois de la langue française (OQLF) ${ }^{45}$. L'internaute a ainsi accès au Vocabulaire de l'informatique et de l'Internet, publié dans la Gazette officielle du Québec, qui présente les termes officiels, dont l'emploi est obligatoire ${ }^{46}$, comme en France. Outre ces banques de données de l'OQLF, l'usager peut consulter différents dictionnaires sur Internet ${ }^{47}$.

Malgré l'intérêt commun du Québec et de la France à renforcer la présence du français sur Internet, on remarque des divergences par rapport à la terminologie officielle entre la langue hexagonale et celle du Québec $^{48}$. Le tableau 4 en présente quelques exemples ${ }^{49}$.

Pour un Québécois - contrairement à un Français -, l'utilisation du terme "Web" est la norme. Alors qu'un internaute de France suivant le bon usage d'Internet participe à une causette, celui du Québec clavarde. Il est évident que, des deux côtés de l'Atlantique, la terminologie française de la Toile est différente. Un internaute qui utilise un moteur de recherche ne distingue pas forcément les sites de langue française selon qu'ils proviennent de la France ou du Québec. Cette réalité conduit à une influence mutuelle, qui contribue sans doute à l'usage d'un anglicisme comme le Web.

45. http://www.olf.gouv.qc.ca

46. Voir Hélène Cajolet-Laganière et Pierre Martel, La qualité de la langue au Québec (Diagnostic 18), Québec, IQRC et la Commission des états généraux, op. cit., 2001.

47. http://www.spl.gouv.qc.ca/langue/tablematiere2.html, 21 janvier 2005; www.tlfq.ulaval.ca ; www.tlfq.ulaval.ca/axl/index.shtml ; www.usherbrooke.ca, 21 janvier 2005.

48. DGLFLF, Office québécois de la langue française, www.olf.gouv.qc.ca

49. Le nouveau grand dictionnaire terminologique, Journal Officiel (DGLFLF). http://www.granddictionnaire.com/btml/fra/r_motclef/index1024_1.asp ; Journal Officiel (DGLFLF), http://www.journal-officiel.gouv.fr/accueil.php. 
TABLEAU 4

\begin{tabular}{|c|c|c|c|}
\hline Terme anglais & $\begin{array}{c}\text { Terminologie } \\
\text { officielle (France) }\end{array}$ & \begin{tabular}{|c|} 
Usage sur la Toile \\
(non conforme \\
à au moins une \\
des deux \\
terminologies \\
officielles)
\end{tabular} & $\begin{array}{c}\text { Terminologie } \\
\text { officielle (Québec) }\end{array}$ \\
\hline chat & causette & cbat & $\begin{array}{l}\text { clavardage, } \\
\text { bavardage }\end{array}$ \\
\hline newsgroup & forum & forum & $\begin{array}{l}\text { groupe de } \\
\text { nouvelles, } \\
\text { groupe de } \\
\text { discussion, } \\
\text { forum de } \\
\text { discussion }\end{array}$ \\
\hline firewall & $\begin{array}{l}\text { barrière de sécurité } \\
\text { pare-feu }\end{array}$ & & $\begin{array}{l}\text { serveur de filtrage, } \\
\text { adaptatif, coupe- } \\
\text { feu personnel, } \\
\text { pare-feu personnel. }\end{array}$ \\
\hline World Wide Web & $\begin{array}{l}\text { Toile d'araignée } \\
\text { mondiale (T.A.M.), } \\
\text { Toile mondiale, } \\
\text { Toile }\end{array}$ & $\begin{array}{l}\text { Web, w3, www } \\
\text { ouèbe }\end{array}$ & $\begin{array}{l}\text { Web } \\
\text { w3 } \\
\text { www } \\
\text { hyperToile } \\
\text { TAM } \\
\text { Toile } \\
\text { Toile mondiale } \\
\text { Toile d'araignée } \\
\text { mondiale } \\
\text { ouébe } \\
\text { ouaibe }\end{array}$ \\
\hline
\end{tabular}

L'existence de formes concurrentes d'un même terme n'est donc pas due uniquement au contact du français avec l'anglais, mais aussi à la tentative de standardisation, qui fait la promotion de deux termes officiels différents. Il existe une concurrence au sein même de la francophonie. Mais, grâce à l'utilisation qu'en font les internautes, certains termes se répandent plus que d'autres dans la langue courante. De cette manière, ils contribuent à établir un usage plus clair et homogène, et finissent par créer une norme. 
Cette évolution s'est manifestée ces derniers temps en France ${ }^{50}$. De Broglie constate par exemple en 2002 que le mot " courriel ", proposé par le Québec pour remplacer "e-mail", se répand de plus en plus en France. Ce terme a depuis été adopté

et publié par la Commission générale de terminologie et de néologie au Journal officiel du 20 juin 2003. Par cette publication, la Commission générale a souhaité mettre un terme à une période d'hésitation. Utilisé depuis un certain temps, notamment au Québec, ce néologisme s'est progressivement répandu dans l'usage français pour désigner le courrier électronique, qu'il s'agisse, le plus souvent, du message lui-même, ou, par extension, de la messagerie utilisée ${ }^{51}$.

Le français courant sur la Toile témoigne encore d'une influence de la créativité lexicale des internautes sur le lexique. Ceux-ci emploient par exemple " $t$ chatche " et "ouébe " au lieu de " causette ", "chat "et "Web" Le contenu de l'entrée "tchatche - "(de l'esp. chacharear) FAM. 1. Disposition à s'exprimer facilement, à parler beaucoup [...] 2. Langage argotique des cités (verlan, etc.) ${ }^{52}$ " - correspond au sens du terme "chat ", sauf qu'on se sert dans ce dernier cas d'Internet comme moyen de communication. Il fait aussi allusion au langage des jeunes, qui participent en grand nombre à de telles communications. L'internaute peut donc considérer le "tchatche" comme le plus approprié pour désigner ce type de conversation, contribuant ainsi à l'enrichissement sémantique d'un mot existant depuis 1959 dans la langue française. Ce comportement langagier de l'internaute témoigne d'une sensibilité linguistique évidente.

"Ouèbe " et "wouebe", par contre, constituent des adaptations phonétiques sur le plan orthographique ${ }^{53}$. Toutefois, on pourrait dire

50. Xavier Bihan, op. cit.

51. DGLFLF, www.culture.fr/culture/dglf/garde.htm

52. Le petit Robert, op. cit., 2573a. Voir aussi : Tchatche.com (www.tchatche.com), Bienvenue sur Yahoo! Tchatche (fr.chat.yahoo.com).

53. En voici un exemple : "ouèbe. n.m. [WEB] Le web, dans une version francisée se voulant hilarante mais finalement assez lourdingue à la longue. Article lié à celui-ci : vèbe * (www.linux-france.org/prj/jargonf/O/ouegrbe.html). 
qu'il s'agit, ici de graphies utilisées sur des pages personnelles, qui "peuvent apporter une touche d'originalité ou d'humour aux yeux des auteurs, mais [qui] ne possèdent pas les qualités d'une dénomination ${ }^{54}$. Mais l'usage croissant de "ouèbe "sur la Toile en France (voir tableau 3) doit être considéré. Cette utilisation pourrait être favorisée par le langage du chat, où l'orthographe reflète souvent la prononciation. L'avenir nous dira à quel point ces formes se répandront et, par conséquent, si elles seront lexicalisées et admises dans le bon usage de la Toile et dans le langage quotidien des internautes.

On constate que le lexique utilisé sur Internet est caractérisé par une concurrence non seulement du français avec l'anglais, mais aussi à l'intérieur de la France ou de la francophonie. Il ne fait pas de doute que la langue s'enrichit grâce aux mesures législatives, mais elle le fait aussi par l'usage particulier de ses locuteurs.

L'usage d'Internet témoigne en effet d'une sensibilité linguistique des internautes francophones en faveur de leur idiome maternel, par exemple lorsqu'ils rejettent certains termes officiels au profit d'autres lemmes français. On pourrait alors affirmer, suivant Vaugelas, que "c'est l'usage qu'on appelle le Maistre des langues ${ }^{55}$. Le sentiment naturel des internautes français aboutit finalement à une sélection qui, comme nous l'avons déjà mentionné, peut contribuer à rendre la langue plus claire et plus pure, un aspect souvent négligé dans les discussions concernant l'américanisation du français sur Internet ${ }^{56}$.

On peut conclure que la protection du français, en France comme au Québec, dans le but de consolider sa présence sur le réseau mondial, contribue aussi à créer une certaine hétérogénéité.

La progression du français - et la régression simultanée de l'anglais sur Internet ne témoigne pas seulement de l'effet de la politique linguistique et de la vitalité des internautes francophones naviguant sur la Toile,

54. DGLFLF, Office québécois de la langue française, op. cit.

55. Lothar Wolf, "Texte und Dokumente zur französischen Sprachgeschichte ", Sammlung romanischer Übungstexte, Jahrhundert, Niemeyer, p. 20.

56. Henriette Walter, op. cit. 


\section{REVUE INTERNATIONALE D'ÉTUDES QUÉBÉCOISES}

mais également de leur sensibilité envers leur langue maternelle. L'avenir de la langue française sur Internet ne repose donc pas uniquement sur l'établissement d'une terminologie officielle, mais aussi sur des formes nouvelles provenant de l'usage particulier des internautes, un usage qui prouve l'évolution naturelle et la vitalité de la langue française des deux côtés de l'Atlantique. 